\title{
Hydrogen Bonding versus Entropy: Revealing the Underlying Thermodynamics of the Hybrid Organiclnorganic Perovskite [CH3NH3]PbBr3
} DOI:

10.1021/acs.chemmater.8b03164

\section{Document Version}

Accepted author manuscript

Link to publication record in Manchester Research Explorer

Citation for published version (APA):

Kieslich, G., Skelton, J., Armstrong, J., Wu, Y., Wei, F., Svane, K., Walsh, A., \& Butler, K. (2018). Hydrogen Bonding versus Entropy: Revealing the Underlying Thermodynamics of the Hybrid Organiclnorganic Perovskite $\left[\mathrm{CH}_{3} \mathrm{NH}_{3}\right] \mathrm{PbBr}_{3}$. Chemistry of Materials. https://doi.org/10.1021/acs.chemmater.8b03164

\section{Published in:}

Chemistry of Materials

\section{Citing this paper}

Please note that where the full-text provided on Manchester Research Explorer is the Author Accepted Manuscript or Proof version this may differ from the final Published version. If citing, it is advised that you check and use the publisher's definitive version.

\section{General rights}

Copyright and moral rights for the publications made accessible in the Research Explorer are retained by the authors and/or other copyright owners and it is a condition of accessing publications that users recognise and abide by the legal requirements associated with these rights.

\section{Takedown policy}

If you believe that this document breaches copyright please refer to the University of Manchester's Takedown Procedures [http://man.ac.uk/04Y6Bo] or contact uml.scholarlycommunications@manchester.ac.uk providing relevant details, so we can investigate your claim.

\section{OPEN ACCESS}




\title{
Hydrogen bonding versus entropy: revealing the underlying thermodynamics of the hybrid organic-inorganic perovskite $\left[\mathrm{CH}_{3} \mathrm{NH}_{3}\right] \mathrm{PbBr}_{3}$
}

\author{
Gregor Kieslich, ${ }^{*, \dagger}$ Jonathan Michael Skelton, ${ }^{\ddagger}$ Jeff Armstrong, Yue Wu, \\ Fengxia Wei, ${ }^{\S}$ Katrine Louise Svane, ${ }^{\ddagger}$ Aron Walsh, ${ }^{\prime}$ and Keith T. Butler, \\ $\dagger$ Department of Chemistry, Technical University of Munich, Lichtenbergstraße 4, 85748 \\ Garching, Germany \\ $\ddagger$ Department of Chemistry, University of Bath, Claverton Down, Bath BA2 7AY, UK \\ ฯISIS Facility, Rutherford Appleton Laboratory, Harwell Oxford, Didcot, Oxfordshire OX11 \\ $O Q X, U K$ \\ $\S$ Department of Materials Science and Metallurgy, University of Cambridge, 27 Charles \\ Babbage Road, Cambridge CB3 OFS, UK \\ ||Department of Materials, Imperial College London, Royal School of Mines, Exhibition \\ Road, London, SW7 2AZ, UK
}

E-mail: gregor.kieslich@tum.de; keith.butler@stfc.ac.uk

\begin{abstract}
The enormous research efforts on hybrid organicinorganic perovskites have led to a deep understanding of these materials; however, the role of entropy and its ramifications on the materials properties have only been sparsely explored. In this study we quantify the phase transition mechanism in the hybrid organicinorganic perovskite $\left[\mathrm{CH}_{3} \mathrm{NH}_{3}\right] \mathrm{PbBr}_{3}$ by studying low energy collective phonon modes using a combination of inelastic neutron scattering and $a b$ initio lattice dynamics. We demonstrate that a delicate interplay between hydrogen bonding interactions, lattice vibrational entropy and configurational disorder determines the thermodynamics and results in the rich phase evolution of $\left[\mathrm{CH}_{3} \mathrm{NH}_{3}\right] \mathrm{PbBr}_{3}$ as a function of temperature. Our results have important implications for the manipulation of macroscopic properties and provide a blueprint for future studies that focus on unravelling phase transition mechanisms in hybrid perovskites and related materials such as dense and porous coordination polymers.
\end{abstract}

\section{Introduction}

Understanding how the chemistry of a system affects the structure and physical properties is at the heart of materials science. Entropy plays a critical role in determining the structural and dynamic properties of materials. ${ }^{1}$ In solid-state inorganic systems the role of configurational disorder has long been recognised in the context of alloys and solid solutions. ${ }^{2-4}$ In biological systems and solutions the contribution of vibrational entropy has been shown to be of the utmost importance in driving phase equilibria. ${ }^{5,6}$ Glass forming behaviour is dependent on the combination of vibrational and configurational entropy. ${ }^{7-9}$ In the context of hybrid organic-inorganic perovskites (HOIPs), we have recently shown computationally that vibrational entropy can drive phase transitions in materials such as $\left[\mathrm{CH}_{3} \mathrm{NH}_{3}\right] \mathrm{PbI}_{3}$ or $\left[\mathrm{NH}_{3} \mathrm{NH}_{2}\right] \mathrm{Zn}(\mathrm{HCOO})_{3} \cdot{ }^{10-12}$

HOIPs have risen to prominence in recent years in fields such as photovoltaics and light emitting diodes. ${ }^{13}$ In photovoltaics the per- 
ovskites have revolutionised the field, offering low cost, high-efficiency devices after only a few years of development. ${ }^{14,15}$ Even in the relatively short time that there has been active research on HOIPs, it has become apparent that low frequency modes (up to around $5 \mathrm{THz}$ ), configurational disorder and hydrogen bonding are critical in a variety of ways such as the phase stability and electron-phonon coupling. ${ }^{16}$ Inelastic and quasielastic scattering studies on HOIPs have revealed fascinating insights, such as soft phonon modes and local symmetry breaking phenomena. ${ }^{17-19}$ However, a deeper understanding of low frequency lattice dynamics modes, molecular disorder, intermolecular bonds and their link to macroscopic properties is required to allow for rational materials design principles to be developed.

Understanding low frequency modes is as challenging as it is important. Beyond vibrational entropy they have been implicated in a wide range of important physical phenomena such as thermal conductivity, ${ }^{20}$ negative thermal expansion, ${ }^{21,22}$ mechanical instabilities, ${ }^{23-25}$ co-crystallisation ${ }^{26}$ and structural destabilisation. $^{27}$ In recent years the combination of inelastic neutron spectroscopy (INS) with $a b$ initio lattice dynamics has provided powerful insights into the mechanisms involved in low frequency vibrations of solid-state ${ }^{28,29}$ and hybrid materials. ${ }^{30-35}$ In particular, INS studies have helped to develop a deeper understanding of the motions of molecules and framework in the halide perovskites across phase transitions. ${ }^{36,37}$ Lattice dynamics calculations performed using density functional theory (DFT) can be used to calculate the vibrational spectrum of a material. The calculated vibrational spectrum can then be related to the experimental INS spectrum, which allows for a deconvolution of the complex manifold of phonons involved in low-frequency vibrations into characteristic molecular and atomic motions. For instance, this type of analysis has recently been used for the exploration of the role of low frequency vibrations in phenomena such as gate-opening and mechanical shearing in metal-organic frameworks. ${ }^{38}$

In this study we combine terahertz $(\mathrm{THz})$
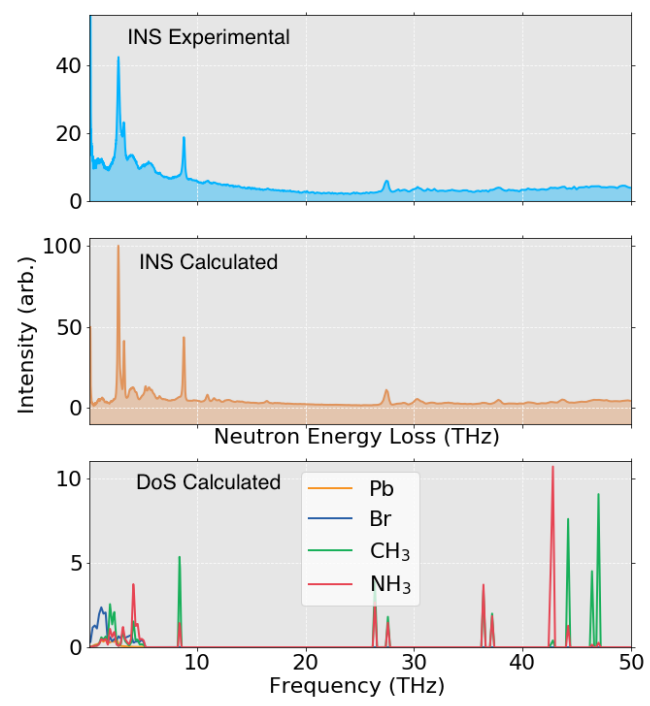

Figure 1: A comparison of the INS (Top) and $a b$ initio calculated (middle) neutron energy loss spectra, with the partial density of states (bottom). The INS spectrum was collected on the TOSCA beamline at $10 \mathrm{~K}$.

INS, with DFT lattice dynamics to explore the origin and ramifications of entropic effects in the halide perovskite $\left[\mathrm{CH}_{3} \mathrm{NH}_{3}\right] \mathrm{PbBr}_{3}$. After confirming our structural model by comparing the experimental INS data with our calculations, we analyse the role and aspects of entropy and hydrogen bonding interactions in determining the thermodynamics of $\left[\mathrm{CH}_{3} \mathrm{NH}_{3}\right] \mathrm{PbBr}_{3}$. Finally, we link the microscopic details of the vibrational modes to the differences in structure and ultimately phase stability between the orthorhombic, tetragonal and cubic polymorphs of $\left[\mathrm{CH}_{3} \mathrm{NH}_{3}\right] \mathrm{PbBr}_{3}$. The quantitative picture we obtain then allows us to discuss potential design principles that can manipulate underlying thermodynamics and in turn macroscopic properties in these materials.

\section{Low frequency vibrations}

High-resolution INS measurements were performed on powdered $\left[\mathrm{CH}_{3} \mathrm{NH}_{3}\right] \mathrm{PbBr}_{3}$ at approximately $10 \mathrm{~K}$, using the inverted-geometry neutron spectrometer TOSCA1-3 located at the 
ISIS Pulsed Neutron \& Muon Source, Rutherford Appleton Laboratory, UK, see electronic supplementary information (ESI) for details. The experimental INS data is presented in Figure 1a. Above $12 \mathrm{THz}$, the spectral intensities fall away with increased energy transfer due to the presence of phonon wings. ${ }^{39}$ In the low energy region of the spectrum a number of sharp peaks are observed. These peaks are associated with the motion of the $\left[\mathrm{CH}_{3} \mathrm{NH}_{3}\right]^{+}$molecule in $\left[\mathrm{CH}_{3} \mathrm{NH}_{3}\right] \mathrm{PbBr}_{3}$ and are pronounced due to the large incoherent neutron scattering crosssection of hydrogen. Notably, these signals have a similar energy and envelope to those observed previously in $\left[\mathrm{CH}_{3} \mathrm{NH}_{3}\right] \mathrm{PbI}_{3},{ }^{40}$ indicating that the molecule in $\left[\mathrm{CH}_{3} \mathrm{NH}_{3}\right] \mathrm{PbBr}_{3}$ is also not fully frozen, even at low temperatures.

For a quantitative interpretation of the obtained INS spectra we compare our results to $a b$ initio lattice dynamics calculations. The phonon spectrum of $\left[\mathrm{CH}_{3} \mathrm{NH}_{3}\right] \mathrm{PbBr}_{3}$ in the orthorhombic phase was calculated using the frozen phonon approximation. Energies and forces of the system were calculated from the generalised gradient approximations (GGA) of DFT, using the VASP package. The dynamical matrix and phonon frequencies are obtained using the PHONOPYpackage. The calculated phonon spectrum was converted to an INS spectrum using the semi-empirical approach developed by Howard and co-workers and as implemented in the latest version of the MANTID package, ${ }^{41,42}$ this approach considers the vibrational spectrum across all of $Q$-space. The simulated INS spectrum thus obtained is shown in Figure 1b. The simulated spectrum from ab initio lattice dynamics is in excellent agreement with the experimental spectrum. It has previously been shown that INS spectra are a highly sensitive probe of structure, and a powerful tool for ascertaining the validity of a model system, being able to discriminate between small changes to local environment. Thus the match between experimental and calculated spectra gives us high confidence in our structural model of the orthorhombic ground state of $\left[\mathrm{CH}_{3} \mathrm{NH}_{3}\right] \mathrm{PbBr}_{3} .{ }^{43}$ Full details of the computational set up are available in the ESI.

From the lattice dynamics calculations the nature of the vibrations responsible for the neutron energy loss can be explored in more detail. At very low frequencies up to $1.5 \mathrm{THz}$, the modes in orthorhombic $\left[\mathrm{CH}_{3} \mathrm{NH}_{3}\right] \mathrm{PbBr}_{3}$ are mainly associated with the $\mathrm{Br}$ atoms, see partial DOS in Figure 1. These modes are best described as typical perovskite-tilting modes, in which $\mathrm{PbBr}_{6}$ octahedra tilt around the centre, although we note that some of these modes also involve breathing of the octahedra (see our online repository, eg mode $7^{1}$ ). Between 1.5 - $2.7 \mathrm{THz}$ the modes involve concerted motion of both the $\left[\mathrm{CH}_{3} \mathrm{NH}_{3}\right]^{+}$cation and the cages, with vibrations of $\left[\mathrm{CH}_{3} \mathrm{NH}_{3}\right]^{+}$coupled to the tilting of the $\mathrm{PbBr}_{6}$ octahedra. At 2.7 $\mathrm{THz}$ a clear peak associated with motion of $\left[\mathrm{CH}_{3} \mathrm{NH}_{3}\right]^{+}$can be observed, i.e. a nodding motion of the $\mathrm{CH}_{3}$ end of $\left[\mathrm{CH}_{3} \mathrm{NH}_{3}\right]^{+}$which has been described previously as the "nodding donkey" mode. ${ }^{40}$ The modes at $4.5 \mathrm{THz}$ arise from several types of vibrations, e.g. torsional motions of $\left[\mathrm{CH}_{3} \mathrm{NH}_{3}\right]^{+}$in which the $\mathrm{CH}_{3}$ and $\mathrm{NH}_{3}$ ends twist in opposite directions, librational modes of $\left[\mathrm{CH}_{3} \mathrm{NH}_{3}\right]^{+}$and modes associated with breathing motions of the inorganic $\left[\mathrm{PbBr}_{3}\right]^{-}$lattice where alternating $\mathrm{PbBr}_{6}$ units expand and contract. Animations of all modes are available in an on-line repository ${ }^{1}$. We also performed similar calculations for the tetragonal and cubic phases of $\left[\mathrm{CH}_{3} \mathrm{NH}_{3}\right] \mathrm{PbBr}_{3}$, revealing that the nature of the modes is largely unchanged between different polymorphs.

Interestingly, the site resolved phonon DOS analysis reveals evidence for $\mathrm{Pb}^{2+} s^{2}$ lone pair activity at low frequencies. To further investigate the possibility of a stereochemically active lone pair we have calculated the electronic structure of the material at the equilibrium position and distorted along the mode at 2.6 THz. Figure 2 shows an isodensity plot of the valence band maximum, arising from $\mathrm{Pb}$ $6 s$ and $\mathrm{Br} 4 p$ orbitals. The $\mathrm{Pb}$ electron density is clearly anisotropically distorted at the extreme of the mode, demonstrating the importance of a stereoactive lone pair in this phonon mode and more generally in the low frequency vibrational behaviour of the mate-

\footnotetext{
${ }^{1}$ https://github.com/keeeto/MAPbBr3-Phonons
} 


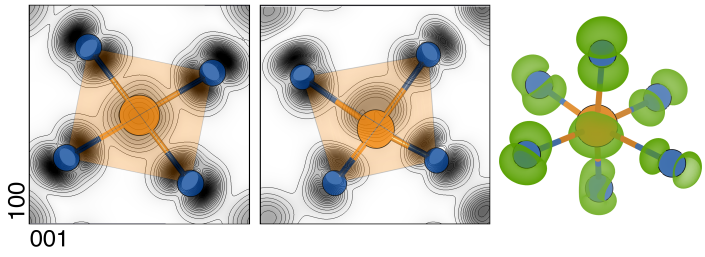

Figure 2: Lone pair activity in lattice vibrations. The equilibrium (left) and extended (centre) configurations of the vibrational mode at $2.6 \mathrm{THz}$. The charge density of the valence band maximum is plotted in the background using an isolevel interval of $3.5 \times 10^{-4}$ e. (Right) A $3-\mathrm{D}$ representation of the same charge density.

rial. This finding is particularly intruiging since experimental evidence for lone pair activity has been discussed for the $\mathrm{Sn}^{2+}$ and $\mathrm{Ge}^{2+}$ homologues e.g. $\left[\mathrm{CH}_{3} \mathrm{NH}_{3}\right] \mathrm{SnBr}_{3}, \mathrm{CsSnBr}_{3}$ and $\left[\mathrm{CH}_{3} \mathrm{NH}_{3}\right] \mathrm{GeBr}_{3} \cdot{ }^{44-46}$

\section{Thermodynamics}

We next consider how the thermal occupation of these modes impacts the thermodynamics of different polymorphs of $\left[\mathrm{CH}_{3} \mathrm{NH}_{3}\right] \mathrm{PbBr}_{3}$. The high density of low energy vibrational modes and their thermal populations provide an important contribution to the Helmholtz free energy of the system in the form of vibrational entropy. The vibrational entropy, which can be calculated from both the experimental and the theoretical DOS by integrating over the DOS with a population factor, provides a bridge between the microscopic details of the crystal and the observed macroscopic properties related to lattice dynamics, e.g. phase stabilities and thermal conductivities. From the phonon DOS $(g(\varepsilon))$, the lattice entropy can be obtained from equation (1),

$$
\begin{array}{r}
S_{v i b}(T)=3 k_{B} \int_{0}^{\infty} g(\varepsilon)[(n(\varepsilon)+1) \operatorname{Ln}(n(\varepsilon)+1) \\
-n(\varepsilon) \operatorname{Ln}(n(\varepsilon))] d \varepsilon
\end{array}
$$

where $n(\varepsilon)=\left(\exp \left[\varepsilon / k_{B} T\right]-1\right)^{-1}, k_{B}$ is Boltzmann's constant and $\varepsilon$ is the mode energy.
The vibrational entropy as a function of temperature is shown in Figure 3 inset (full range in the ESI). Experimentally it has been established that $\left[\mathrm{CH}_{3} \mathrm{NH}_{3}\right] \mathrm{PbBr}_{3}$ crystallises in an orthorhombic phase at low temperatures (Pnma). When increasing the temperature and only considering Bragg scattering, it is observed that $\left[\mathrm{CH}_{3} \mathrm{NH}_{3}\right] \mathrm{PbBr}_{3}$ transforms to a tetragonal-II phase $(\mathrm{P} 4 / \mathrm{mmm})$ at $T=145 \mathrm{~K}$ and further to a tetragonal-I phase (I4/ $\mathrm{mcm}$ ) at $T=155 \mathrm{~K}$. At temperatures above $237 \mathrm{~K}$ $\left[\mathrm{CH}_{3} \mathrm{NH}_{3}\right] \mathrm{PbBr}_{3}$ becomes cubic $(\mathrm{Pm} \overline{3} \mathrm{~m})$ with a highly dynamic $\left[\mathrm{PbBr}_{3}\right]^{-}$framework. ${ }^{47-49}$ To gain insight into the contributions of vibrational entropy to the different phase transitions, whilst maintaining a reasonable level of computational resource we consider three phases; $\mathrm{S}_{\text {vib }}^{\text {ortho }}(\mathrm{T})$ is compared with $\mathrm{S}_{\text {vib }}^{\text {tet }}(\mathrm{T})$ (tetragonalII phase) and $\mathrm{S}_{v i b}^{c u b i c}(\mathrm{~T})$. Notably, the vibrational entropies as a function of temperature were obtained by applying the same methodology as for the orthorhombic phase. We discuss the origins of configurational entropy in more detail in the ESI.

The vibrational entropies $\left(S_{v i b}\right)$ of the tetragonal and the orthorhombic phases are nearly identical across the whole temperature range (the orthorhombic phase has $0.85 \mathrm{JK}^{-1} \mathrm{~mol}^{-1}$ greater $\mathrm{S}_{v i b}$ ), thus the phase transition from orthorhombic to tetragonal is not driven by gain in vibrational entropy. Instead, as a result of the increased number of available orientations of the $\left[\mathrm{CH}_{3} \mathrm{NH}_{3}\right]^{+}$cation in the tetragonal phase, it is the gain in configurational entropy $\left(S_{\text {config }}\right)$ that shifts the thermodynamics in favour of the tetragonal-II phase. In a simplified picture and under the assumption of no disorder of $\left[\mathrm{CH}_{3} \mathrm{NH}_{3}\right]^{+}$between $\mathrm{C}$ and $\mathrm{N}$, the difference in $S_{\text {config }}$ between the orthorhombic and tetragonal phase is $S_{\text {config }}=R \operatorname{Ln}(4)=11.53$ $\mathrm{JK}^{-1} \mathrm{~mol}{ }^{-1}$ and is independent of temperature. ${ }^{47}$ The total Helmholtz free energy $(F)$ of the system can be calculated from the enthalpy $(H)$, vibrational energy $\left(E_{v i b}\right)$ and the entropy

$$
F=H+E_{v i b}-T\left(S_{\text {config }}+S_{v i b}\right) .
$$

The calculated Helmholtz free energy is plotted in Figure 3 and shows the phase transition from 

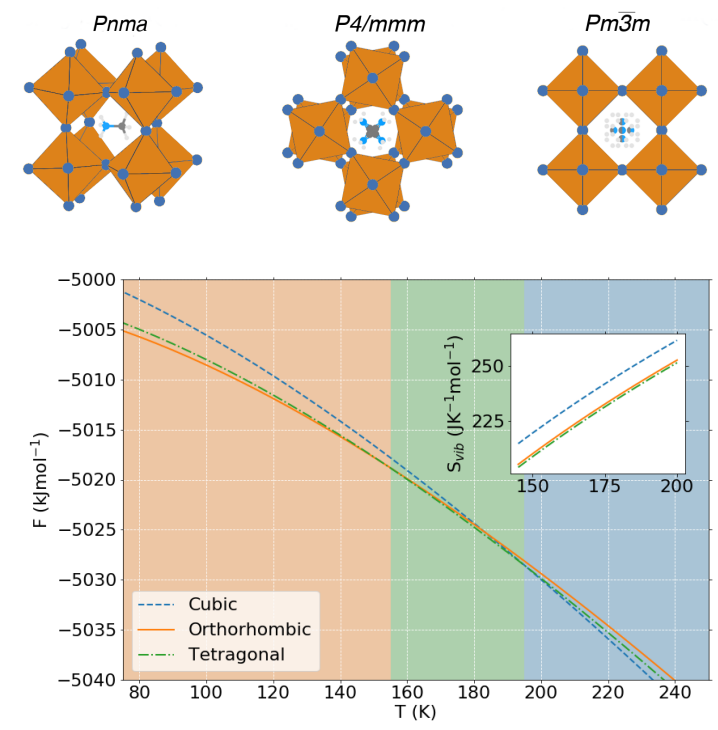

Figure 3: The phases and free energy with temperature. (a) Schematic representations of the three phases considered here, from left to right orthorhombic, tetragonal-II and cubic phases.(b) The total free energy of the three phases calculated according to Equation 2. The regions of stability of the three phases are shaded accordingly. The inset shows the vibrational entropy $\left(\mathrm{JK}^{-} 1 \mathrm{~mol}^{-1}\right)$ versus temperature $(\mathrm{K})$ in the region of the phase transitions.

orthorhombic to tetragonal at $152 \mathrm{~K}$, which is in excellent quantitative agreement with the experimental measurement of $145 \mathrm{~K} .{ }^{47}$

The phase transition from tetragonal to cubic is associated with an increase in both $S_{v i b}$ and $S_{\text {config. }}$ Figure 1 of the ESI and $3 \mathrm{~b}$ inset, shows the difference in $S_{v i b}$ between the tetragonal and cubic phase, which is up to $11.39 \mathrm{JK}^{-1} \mathrm{~mol}^{-1}$ and is around $10 \mathrm{JK}^{-1} \mathrm{~mol}^{-1}$ in the region of the phase transition (see Figure 3 inset). Under the assumption of disorder around the C$\mathrm{N}$ axis in the cubic phase, the gain in $S_{\text {config }}$ from tetragonal-II to cubic can be calculated as $R L n(6) \mathrm{JK}^{-1} \mathrm{~mol}^{-1} \cdot{ }^{47}$ This results in the overall difference of entropy between the tetragonalII and cubic phase of $\Delta \mathrm{S}=\Delta \mathrm{S}_{v i b}+\Delta \mathrm{S}_{\text {config }}$ $=25.58 \mathrm{JK}^{-1} \mathrm{~mol}^{-1}$. This entropic difference, which is greater than that of the orthorhombic to tetragonal transition, outweighs the enthalpy difference between the tetragonal and cubic phases at $197 \mathrm{~K}$, although this is somewhat lower than the experimentally reported transition $(237 \mathrm{~K})$, we note that we consider only one of the two tetragonal phases here.

The thermodynamic properties presented here are calculated on $0 \mathrm{~K}$ ground-state structures, within the harmonic approximation of lattice dynamics. There are two important assumptions that need to be validated. (i) Lattice expansion and anharmonicity are ignored. In the ESI we calculate the mode Grüneisen parameters and estimate the effects of lattice expansion on vibrational frequencies and thermal properties. (ii) Imaginary modes are excluded from the partition function and therefore do not contribute to the vibrational free energy. In the ESI we used the approach outlined in Ref. ${ }^{50}$ to estimate effective (renormalised) harmonic frequencies and an associated correction to the free energy. Both effects are shown to be negligible in the systems studied here, although they have important consequences for mechanisms of phase transition and could be important in the thermodynamics of other materials.

\section{Microscopic origin of thermodynamics}

After establishing the origin of thermodynamic differences, we are now in a position to explore the sources of vibrational entropy for the different polymorphs in more detail. To facilitate this analysis, we calculate the vibrational entropy contributions from the various atomic sites, by integrating the partial DOS for the various sites, equation 3 .

$$
\Delta S(v)=\int_{0}^{v}\left(S_{v i b}^{a}(v)-S_{v i b}^{b}(v)\right) d v
$$

where $v$ is the frequency and superscripts $a$ and $b$ refer to the different polymorphs, with $S_{v i b}$ calculated as in Equation 1. Due to the exponential decay of the Boltzmann population of the vibrational states, the differences in entropy contributions fall off rapidly as $v$ increases, so that almost all of the difference arises from modes with $v<15$ THz. ${ }^{11}$ The obtained differences between the integrated site entropies $(\Delta S)$ of the phases are shown in Table 1.

Although the total $S_{v i b}$ in the orthorhombic 
Table 1: Entropy changes at phase transitions. The difference in site resolved vibrational entropy between the phases involved in the two phase transitions. Differences in $\Delta S_{v i b}$ are reported in $\mathrm{JK}^{-1} \mathrm{~mol}^{-1}$ and $T_{\text {trans }}$ (transition temperature) are in $\mathrm{K}$.

\begin{tabular}{l|ccccc|c}
\hline Transition & $\Delta S_{v i b}^{P b}$ & $\Delta S_{v i b}^{B r}$ & $\Delta S_{v i b}^{N H_{3}}$ & $\Delta S_{v i b}^{C H_{3}}$ & $\Delta S_{v i b}^{\text {Tot }}$ & $\mathrm{T}_{\text {trans }}$ \\
\hline $\begin{array}{l}\text { Orthorhombic } \\
\rightarrow \text { Tetragonal }\end{array}$ & -0.18 & -0.58 & -1.49 & 1.39 & -0.86 & 152 \\
$\begin{array}{l}\text { Tetragonal } \\
\rightarrow \text { Cubic }\end{array}$ & -1.01 & 7.14 & 5.57 & -1.01 & 10.68 & 197 \\
\hline \hline
\end{tabular}

and tetragonal phases are similar, the site contributions to entropy are significantly different, see Table 1. The $\mathrm{NH}_{3}$ moiety contributes more in the orthorhombic phase and the $\mathrm{CH}_{3}$ moiety has larger contributions in the tetragonal phase. Interestingly, the $\mathrm{Br} S_{v i b}$ contribution decreases slightly across the phase transition, which implies a more rigid framework in the tetragonal phase. This is contrary to the intuitive picture, where the framework should become more flexible with increasing temperature. However, the increased $S_{\text {config }}$ is sufficient to favour this unexpected result. We note that there is an additional tetragonal phase reported experimentally $(\mathrm{I} 4 / \mathrm{mcm}),{ }^{40,51}$ the disorder in the $\mathrm{I} 4 / \mathrm{mcm}$ phase is greater, by a factor of 2 . However the binding of the molecule seems to be similar, in both phases it has been reported ${ }^{52}$ that the $\mathrm{CH}_{3}$ moiety is not hydrogen bonded in the $\mathrm{I} 4 / \mathrm{mcm}$ phase as in our model $\mathrm{P} 4 / \mathrm{mmm}$ system.

The difference in the site contributions to $S_{v i b}$ between the tetragonal and cubic phases is shown in Table 1 . In this case there is a pronounced difference between the total $S_{v i b}$ of the two phases, which favours the cubic polymorph at higher temperatures. In terms of $S_{v i b}$ arising from the $\left[\mathrm{CH}_{3} \mathrm{NH}_{3}\right]^{+}$cation, the cubic phase essentially recovers the vibrational entropy forfeited in the orthorhombic to tetragonal transition at the $\mathrm{NH}_{3}$ end, whilst forfeiting some of the $S_{v i b}$ from the $\mathrm{CH}_{3}$ end; a situation that corresponds to the $\left[\mathrm{CH}_{3} \mathrm{NH}_{3}\right]^{+}$cation being overall more loosely bound to the $\left[\mathrm{PbBr}_{3}\right]^{-}$cage in the cubic phase than either of the other phases. The average $\mathrm{CH}_{3}$-Br separation is $3.36 \AA$ and the average $\mathrm{NH}_{3}$ - $\mathrm{Br}$ separation is $2.92 \AA$ in the cubic polymorph compared to $3.22 \AA$ and 2.83 $\AA$ respectively in the tetragonal polymorph, note that these values are calculated from DFT at $0 \mathrm{~K}$ and as such are merely indicative of tighter bonding, but should not be treated as quantitative representations of the real structure, where the bonds will have large thermal motions. In addition to the increased $S_{v i b}$ from $\left[\mathrm{CH}_{3} \mathrm{NH}_{3}\right]^{+}$, there is also a large increase in $S_{v i b}$ from the $\mathrm{Br}$ atoms in the cubic phase compared to tetragonal. ${ }^{53}$ This corresponds to a wider range of tilting in the $\mathrm{PbBr}_{6}$ octahedra and again is a result of the less dense, more open structure of the cubic phase compared to the other phases.

The results that we have presented fit into a wider picture that is emerging related to phase changes in hybrid halide perovskites. Guo and co workers demonstrated that there is a coupling between the dynamics of organic and inorganic components, this results in an abrupt phase transition, when hydrogen bonding is broken, and is contrasted to the gradual phase transition in the all inorganic $\mathrm{CsPbBr}_{3} .{ }^{51}$ Their findings are in line with neutron scattering studies of the phase transition, which find a band of vibrations at around $5 \mathrm{meV}$ which shows soft modes at the phase transition, ${ }^{36}$ and Raman studies that show hydrogen bonding affects octahedral tilting. ${ }^{52}$ The overall picture of the phase transition that emerges from these studies is of a mixed displacive/orderdisorder transition. Both of these studies emphasise the importance of hydrogen bonding in these systems, as do our results. Previously Swainson and co-workers showed that the $\mathrm{NH}_{3}$ 
- Br hydrogen bond affects the lone pair activity of $\mathrm{Sn}$ in $\left[\mathrm{CH}_{3} \mathrm{NH}_{3}\right] \mathrm{SnBr}_{3} .{ }^{45}$ In light of the lone pair activity that we demonstrate here, this kind of interaction may also be important in B-site off-centring in $\mathrm{Pb}$ halide perovskites. This kind of tuning of lone pair activity has been demonstrated on all-inorganic halide perovskites, where larger A-sites, lighter B-sites and harder X- sites all drive increased lone pair activity. ${ }^{44,46,54}$ Increased lone pair activity then has applicational implications, affecting properties such as lattice polarisation and charge carrier lifetimes. Tuning the the hydrogen bonding interactions between molecule and framework, by balancing the enthalpy and vibrational entropy of the interactions, provides an additional handle for altering the phase transition and structural properties of hybrid halide perovskites.

\section{Conclusions}

In conclusion, we unravelled and quantified the thermodynamic origin of the phase evolution of the hybrid organic-inorganic perovskite $\left[\mathrm{CH}_{3} \mathrm{NH}_{3}\right] \mathrm{PbBr}_{3}$ by combining high resolution inelastic neutron scattering with $a b$ initio lattice dynamics. The differences in enthalpy, configurational and vibrational entropy of the phases are all of the same order, meaning that each of these factors are important in determining the stable structure as a function of temperature. These contributions are even more significant, when one considers the decreased role of the Madelung energy in determining structure in halide perovskites, comapred to oxides. ${ }^{55} \mathrm{We}$ find that a complex interplay between entropy and hydrogen bonding interactions leads to the phase transitions as a function of temperature; a result that seems to be generally applicable for crystalline hybrid materials. These insights can now be used to understand behaviours in related materials and for the design of materials with tunable properties. For instance, it seems that by small substitutions of $\mathrm{Pb}^{2+}$ with $\mathrm{Sn}^{2+}$ or $\mathrm{Ge}^{2+}$, low frequency modes related to the stereoactive lone pair can be directly manipulated. Likewise, lattice dynamics related to hydrogen bonding strength play an important role and can be manipulated by replacing $\left[\mathrm{CH}_{3} \mathrm{NH}_{3}\right]^{+}$with $\left[\left(\mathrm{NH}_{2}\right)_{2} \mathrm{CH}\right]^{+}$. Such attempts, however, also go beyond these considerations e.g. the size of the cation must be considered which in turn can have a significant impact on the crystal chemistry and the symmetry of the molecule can have an impact on configurational entropy. More generally, the methodology applied here demonstrates the power of $a b$ initio lattice dynamics to provide predictions in excellent agreement with experiment. The combination of INS with $a b$ initio calculations provides a blueprint of how to understand lattice vibrations and relate them to macroscopic properties in an accurate, quantitative way, not only in the context of hybrid organic-inorganic perovskites, but similarly for related hybrid materials. For instance, in flexible metal-organic frameworks, a balance between enthalpic dispersion forces and vibrational entropy exists; our methodology paves the way to understand these on a microscopic level and in turn facilitates the targeted design of such effects. ${ }^{56}$

We acknowledge membership of the UK's HPC Materials Chemistry Consortium (EPSRC EP/L000202) and access to computational resources through PRACE. A.W. acknowledges support from the Royal Society for a University Research Fellowship and K.T.B. is funded by EPSRC (EP/M009580/1 and EP/J017361/1). GK acknowledges the 'Fonds der chemischen Industrie' for support through the Liebig fellowship scheme. The authors also would like to thank Prof. A. K. Cheetham for illuminating discussions. We thank H. Boström and E. Reynolds for help with collecting INS data.

Data analysis scripts used to generate Figures 2-4, the optimised structures, and data from the phonon calculations are available online, free of charge, from https://github.com/keeeto/MAPbBr3Phonons

Supporting information. Vibrational entropy plotted. Description of the origins of configurational entropy. Description of calculations of hydrogen bond strength. Methods for, and results of, calculation of thermal expansion and anharmonicity. Methods for, and results of, cal- 
culation of imaginary modes. Technical details of all calculation setups. Synthesis description. Characterisation (X-ray diffraction and inelastic neutron scattering) details.

\section{References}

(1) Gibbs, J. W. On the Equilibrium of Heterogeneous Substances. Transactions of the Connecticut Academy of Arts and Sciences 1879, 3, $1874-1878$.

(2) Wei, S.-H.; Ferreira, L. G.; Zunger, A. First-principles calculation of temperature-composition phase diagrams of semiconductor alloys. Phys. Rev. B 1990, 41, 8240-8269.

(3) Gludovatz, B.; Hohenwarter, A.; Catoor, D.; Chang, E. H.; George, E. P.; Ritchie, R. O. A fracture-resistant highentropy alloy for cryogenic applications. Science 2014, 345, 1153-1158.

(4) Caetano, C.; Butler, K. T.; Walsh, A. Analysis of electrostatic stability and ordering in quaternary perovskite solid solutions. Phys. Rev. B 2016, 93, 144205.

(5) Dunitz, J. D. Win some, lose some: enthalpy-entropy compensation in weak intermolecular interactions. Chemistry and Biology 1995, 2, 709-12.

(6) Ruggiero, M. T.; Zhang, W.; Bond, A. D.; Mittleman, D. M.; Zeitler, J. A. Uncovering the Connection Between LowFrequency Dynamics and Phase Transformation Phenomena in Molecular Solids. Phys. Rev. Lett. 2018, 120, 196002.

(7) Phillips, J. Topology of covalent noncrystalline solids I: Short-range order in chalcogenide alloys. J. Non-Cryst. Sol. 1979, 34, $153-181$.

(8) Sastry, S. The relationship between fragility, configurational entropy and the potential energy landscape of glassforming liquids. Nature 2001, 409, 164 167.
(9) Martinez, L. M.; Angell, C. A. A thermodynamic connection to the fragility of glass-forming liquids. Nature 2001, 410, 663-667.

(10) Butler, K. T.; Walsh, A.; Cheetham, A. K.; Kieslich, G. Organised chaos: entropy in hybrid inorganicorganic systems and other materials. Chem. Sci. 2016, 7, 6316-6324.

(11) Butler, K. T.; Svane, K.; Kieslich, G.; Cheetham, A. K.; Walsh, A. Microscopic origin of entropy-driven polymorphism in hybrid organic-inorganic perovskite materials. Phys. Rev. B 2016, 94, 180103.

(12) Wei, W.; Li, W.; Butler, K. T.; Feng, G.; Howard, C. J.; Carpenter, M. A.; Lu, P.; Walsh, A.; Cheetham, A. K. An Unusual Phase Transition Driven by Vibrational Entropy Changes in a Hybrid OrganicInorganic Perovskite. Angew. Chem. Int. Ed. 2018, 57, 8932-8936.

(13) Li, W.; Wang, Z.; Deschler, F.; Gao, S.; Friend, R. H.; Cheetham, A. K. Chemically diverse and multifunctional hybrid organicinorganic perovskites. Nat. Rev. Mater. 2017, 2, 16099.

(14) Kojima, A.; Teshima, K.; Shirai, Y.; Miyasaka, T. Organometal Halide Perovskites as Visible-light Sensitizers for Photovoltaic Cells. J. Am. Chem. Soc. 2009, 131, 6050-6051.

(15) Yang, W. S.; Park, B.-W.; Jung, E. H.; Jeon, N. J.; Kim, Y. C.; Lee, D. U.; Shin, S. S.; Seo, J.; Kim, E. K.; Noh, J. H.; Seok, S. I. Iodide management in formamidinium-lead-halidebased perovskite layers for efficient solar cells. Science 2017, 356, 1376-1379.

(16) Kieslich, G.; Goodwin, A. L. The same and not the same: molecular perovskites and their solid-state analogues. Mater. Horiz. 2017, 4, 362-366.

(17) Beecher, A. N.; Semonin, O. E.; Skelton, J. M.; Frost, J. M.; Terban, M. W.; 
Zhai, H.; Alatas, A.; Owen, J. S.; Walsh, A.; Billinge, S. J. L. Direct Observation of Dynamic Symmetry Breaking above Room Temperature in Methylammonium Lead Iodide Perovskite. ACS Energy Lett. 2016, 1, 880-887.

(18) Brown, K. L.; Parker, S. F.; García, I. R.; Mukhopadhyay, S.; Sakai, V. G.; Stock, C. Molecular orientational melting within a lead-halide octahedron framework: The order-disorder transition in $\mathrm{CH} 3 \mathrm{NH} 3$ PbBr 3. Phys. Rev. B 2017, 96, 174111.

(19) Mozur, E. M.; Maughan, A. E.; Cheng, Y.; Huq, A.; Jalarvo, N.; Daemen, L. L.; Neilson, J. R. Orientational Glass Formation in Substituted Hybrid Perovskites. Chem. Mater. 2017, 1016810177.

(20) Babaei, H.; Wilmer, C. E. Mechanisms of Heat Transfer in Porous Crystals Containing Adsorbed Gases: Applications to Metal-Organic Frameworks. Phys. Rev. Lett. 2016, 116, 025902.

(21) Zhou, W.; Wu, H.; Yildirim, T.; Simpson, J. R.; Walker, A. R. H. Origin of the exceptional negative thermal expansion in metal-organic framework-5 Zn 4 O ( 1 , 4 benzenedicarboxylate) 3. Phys. Rev. B 2008, 78, 054114.

(22) Cockayne, E. Thermodynamics of the Flexible MetalOrganic Framework Material MIL-53(Cr) From First-Principles. J. Phys. Chem. C 2017, 121, 4312-4317.

(23) Bennett, T. D.; Cheetham, A. K.; Fuchs, A. H.; Coudert, F.-X. Interplay between defects, disorder and flexibility in metal-organic frameworks. Nat. Chem. 2016, 9, 11-16.

(24) Ryder, M.; Van de Voorde, B.; Civalleri, B.; Bennett, T. D.; Mukhopadhyay, S.; Cinque, G.; FernandezAlonso, F.; De Vos, D.; Rudić, S.; Tan, J.-C. Detecting Molecular Rotational Dynamics Complementing the Low-Frequency Terahertz Vibrations in a Zirconium-Based Metal-Organic Framework. Phys. Rev. Lett. 2017, 118, 255502 .

(25) Bouëssel du Bourg, L.; Ortiz, A. U.; Boutin, A.; Coudert, F.-X. Thermal and mechanical stability of zeolitic imidazolate frameworks polymorphs. APL Mater. 2014, 2, 124110.

(26) Lien Nguyen, K.; Friščić, T.; Day, G. M.; Gladden, L. F.; Jones, W. Terahertz timedomain spectroscopy and the quantitative monitoring of mechanochemical cocrystal formation. Nat. Mater. 2007, 6, 206-209.

(27) Greaves, G. N. Identifying Vibrations That Destabilize Crystals and Characterize the Glassy State. Science 2005, 308, 1299-1302.

(28) Kugler, M.; Brandl, G.; Waizner, J.; Janoschek, M.; Georgii, R.; Bauer, A.; Seemann, K.; Rosch, A.; Pfleiderer, C.; Böni, P.; Garst, M. Band Structure of Helimagnons in MnSi Resolved by Inelastic Neutron Scattering. Phys. Rev. Lett. 2015, 115, 097203.

(29) Zhang, Q.; Fernandes, R. M.; Lamsal, J.; Yan, J.; Chi, S.; Tucker, G. S.; Pratt, D. K.; Lynn, J. W.; McCallum, R.; Canfield, P. C.; Lograsso, T. A.; Goldman, A. I.; Vaknin, D.; McQueeney, R. J. Neutron-Scattering Measurements of Spin Excitations in $\mathrm{LaFeAsO}$ and $\mathrm{Ba}$ ( Fe 0.953 Co 0.047 ) 2 As 2 : Evidence for a Sharp Enhancement of Spin Fluctuations by Nematic Order. Phys. Rev. Lett. 2015, 114, 057001.

(30) Li, B.; Kawakita, Y.; Liu, Y.; Wang, M.; Matsuura, M.; Shibata, K.; OhiraKawamura, S.; Yamada, T.; Lin, S.; Nakajima, K.; Liu, S. F. Polar rotor scattering as atomic-level origin of low mobility and thermal conductivity of perovskite CH3NH3PbI3. Nat. Commun. 2017, 8, 16086 . 
(31) Pang, J. W. L.; Buyers, W. J. L.; Chernatynskiy, A.; Lumsden, M. D.; Larson, B. C.; Phillpot, S. R. Phonon Lifetime Investigation of Anharmonicity and Thermal Conductivity of UO 2 by Neutron Scattering and Theory. Phys. Rev. Lett. 2013, 110, 157401.

(32) Casco, M. E.; Cheng, Y. Q.; Daemen, L. L.; Fairen-Jimenez, D.; RamosFernández, E. V.; Ramirez-Cuesta, A. J.; Silvestre-Albero, J. Gate-opening effect in ZIF-8: the first experimental proof using inelastic neutron scattering. Chem. Commun. 2016, 52, 3639-3642.

(33) McCabe, E. E.; Stock, C.; Bettis, J. L.; Whangbo, M.-H.; Evans, J. S. O. Magnetism of the Fe $2+$ and $\mathrm{Ce} 3+$ sublattices in Ce 2 O 2 FeSe 2 : A combined neutron powder diffraction, inelastic neutron scattering, and density functional study. Phys. Rev. B 2014, 90, 235115.

(34) Parker, S. F.; Bennington, S. M.; RamirezCuesta, A. J.; Auffermann, G.; Welf, B.; Herman, H.; Williams, K. P. J.; Smith, T. Inelastic Neutron Scattering and Raman Spectroscopies and Periodic DFT Studies of Rb2PtH6 and Rb2PtD6. J. Am. Chem. Soc. 2003, 125, 11656-11661.

(35) Allis, D. G.; Kosmowski, M. E.; Hudson, B. S. The Inelastic Neutron Scattering Spectrum of H3B:NH3 and the Reproduction of Its Solid-State Features by Periodic DFT. J. Am. Chem. Soc. 2004, 126, 7756-7757.

(36) Swainson, I. P.; Stock, C.; Parker, S. F.; Van Eijck, L.; Russina, M.; Taylor, J. W. From soft harmonic phonons to fast relaxational dynamics in $\mathrm{CH}_{3} \mathrm{NH}_{3} \mathrm{PbBr}_{3}$. Phys. Rev. B 2015, 92, 100303.

(37) Létoublon, A.; Paofai, S.; Rufflé, B.; Bourges, P.; Hehlen, B.; Michel, T.; Ecolivet, C.; Durand, O.; Cordier, S.; Katan, C.; Even, J. Elastic Constants, Optical Phonons, and Molecular Relaxations in the High Temperature Plastic Phase of the CH3NH3PbBr3 Hybrid Perovskite. J. Phys. Chem. Lett. 2016, 7, 3776-3784.

(38) Ryder, M.; Civalleri, B.; Bennett, T.; Henke, S.; Rudić, S.; Cinque, G.; Fernandez-Alonso, F.; Tan, J.-C. Identifying the Role of Terahertz Vibrations in Metal-Organic Frameworks: From GateOpening Phenomenon to Shear-Driven Structural Destabilization. Phys. Rev. Lett. 2014, 113, 215502.

(39) Tomkinson, J.; Kearley, G. J. Phonon wings in inelastic neutron scattering spectroscopy: The harmonic approximation. J. Chem. Phys. 1989, 91, 5164-5169.

(40) Leguy, A. M. A.; Goñi, A. R.; Frost, J. M.; Skelton, J.; Brivio, F.; RodríguezMartínez, X.; Weber, O. J.; Pallipurath, A.; Alonso, M. I.; CampoyQuiles, M.; Weller, M. T.; Nelson, J.; Walsh, A.; Barnes, P. R. F. Dynamic disorder, phonon lifetimes, and the assignment of modes to the vibrational spectra of methylammonium lead halide perovskites. Phys. Chem. Chem. Phys. 2016, 18, 27051-27066.

(41) Howard, J.; Boland, B. C.; Tomkinson, J. Intensities in inelastic neutron scattering spectra: A test of recent theory. Chem. Phys. 1983, 77, 145-151.

(42) Arnold, O. et al. MantidData analysis and visualization package for neutron scattering and $\mu \mathrm{SR}$ experiments. Nucl. Instruments Methods Phys. Res. Sect. A Accel. Spectrometers, Detect. Assoc. Equip. 2014, 764, 156-166.

(43) Družbicki, K.; Pinna, R. S.; Rudić, S.; Jura, M.; Gorini, G.; FernandezAlonso, F. Unexpected Cation Dynamics in the Low-Temperature Phase of Methylammonium Lead Iodide: The Need for Improved Models. J. Phys. Chem. Lett. 2016, 7, 4701-4709.

(44) Worhatch, R. J.; Kim, H.; Swainson, I. P.; Yonkeu, A. L.; Billinge, S. 
J. L. Study of Local Structure in Selected Organic-Inorganic Perovskites in the Pm3m Phase. Chem. Mater. 2008, 20, 12721277.

(45) Swainson, I.; Chi, L.; Her, J.-H.; Cranswick, L.; Stephens, P.; Winkler, B.; Wilson, D. J.; Milman, V. Orientational ordering, tilting and lone-pair activity in the perovskite methylammonium tin bromide, CH3NH3SnBr3. Acta Crystallogr. Sect. B Struct. Sci. 2010, 66, 422-429.

(46) Fabini, D. H.; Laurita, G.; Bechtel, J. S.; Stoumpos, C. C.; Evans, H. A.; Kontos, A. G.; Raptis, Y. S.; Falaras, P.; Van der Ven, A.; Kanatzidis, M. G.; Seshadri, R. Dynamic Stereochemical Activity of the Sn2+ Lone Pair in Perovskite CsSnBr3. J. Am. Chem. Soc. 2016, 138, 11820-11832.

(47) Onoda-Yamamuro, N.; Matsuo, T.; Suga, H. Calorimetric and IR spectroscopic studies of phase transitions in methylammonium trihalogenoplumbates (II). J. Phys. Chem. Solids 1990, 51, 1383-1395.

(48) Onoda-Yamamuro N, Matsuo T, S. H. Dielectric study of CH3NH3PbX3 $(\mathrm{X}=\mathrm{Cl}$, Br, I). J. Phys. Chem. Solids 1992, 53, 935-939.

(49) Page, K.; Siewenie, J. E.; Quadrelli, P.; Malavasi, L. Short-Range Order of Methylammonium and Persistence of Distortion at the Local Scale in MAPbBr3 Hybrid Perovskite. Angew. Chem. Int. Edn. 55, 14320-14324.

(50) Skelton, J. M.; Burton, L. A.; Parker, S. C.; Walsh, A.; Kim, C.E.; Soon, A.; Buckeridge, J.; Sokol, A. A.; Catlow, C. R. A.; Togo, A.; Tanaka, I. Anharmonicity in the High-Temperature Cmcm Phase of SnSe: Soft Modes and Three-Phonon Interactions. Phys. Rev. Lett. 2016, 117, 075502.
(51) Guo, Y.; Yaffe, O.; Paley, D. W.; Beecher, A. N.; Hull, T. D.; Szpak, G.; Owen, J. S.; Brus, L. E.; Pimenta, M. A. Interplay between organic cations and inorganic framework and incommensurability in hybrid leadhalide perovskite $\mathrm{CH}_{3} \mathrm{NH}_{3} \mathrm{PbBr}_{3}$. Phys. Rev. Materials 2017, 1, 042401.

(52) Yin, T.; Fang, Y.; Fan, X.; Zhang, B.; Kuo, J.-L.; White, T. J.; Chow, G. M.; Yan, J.; Shen, Z. X. Hydrogen-Bonding Evolution during the Polymorphic Transformations in CH3NH3PbBr3: Experiment and Theory. Chem. Mater. 2017, 29, 5974-5981.

(53) Baikie, T.; Barrow, N. S.; Fang, Y.; Keenan, P. J.; Slater, P. R.; Piltz, R. O.; Gutmann, M.; Mhaisalkar, S. G.; White, T. J. A combined single crystal neutron/X-ray diffraction and solid-state nuclear magnetic resonance study of the hybrid perovskites $\mathrm{CH} 3 \mathrm{NH} 3 \mathrm{PbX} 3$ ( $\mathrm{X}=$ $\mathrm{I}, \mathrm{Br}$ and $\mathrm{Cl}$ ). J. Mater. Chem. A 2015, 3, 9298-9307.

(54) Laurita, G.; Fabini, D. H.; Stoumpos, C. C.; Kanatzidis, M. G.; Seshadri, R. Chemical tuning of dynamic cation off-centering in the cubic phases of hybrid tin and lead halide perovskites. Chem. Sci. 2017, 8, 5628-5635.

(55) Butler, K. T. The chemical forces underlying octahedral tilting in halide perovskites. J. Mater. Chem. C 2018, http://dx.doi.org/10.1039/C8TC02976H, ASAP.

(56) Schneemann, A.; Bon, V.; Schwedler, I.; Senkovska, I.; Kaskel, S.; Fischer, R. A. Flexible metal-organic frameworks. Chem. Soc. Rev. 2014, 43, 6062-6096. 


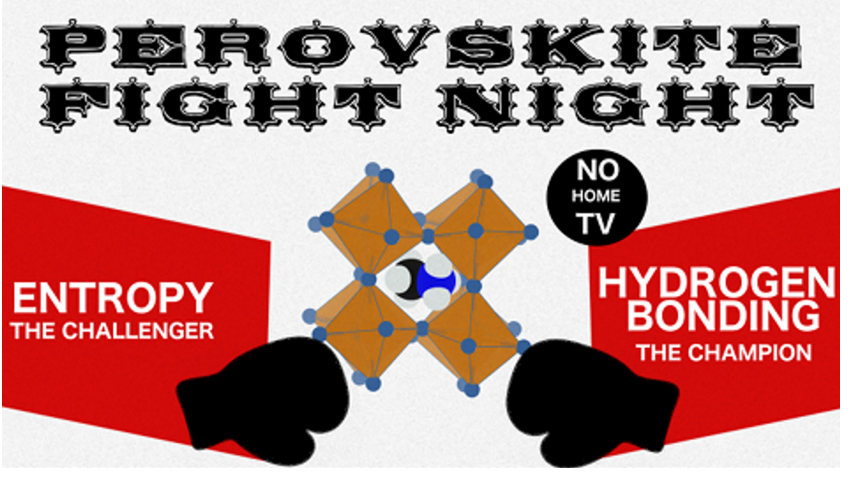

Figure 4: Table of contents figure. 